\title{
Omega-3 polyunsaturated fatty acids prevent murine dilated cardiomyopathy by reducing oxidative stress and cardiomyocyte apoptosis
}

\author{
QIANXIAO LI ${ }^{1}$, QIN YU ${ }^{2}$, RONGMEI NA ${ }^{2}$ and BAITING LIU ${ }^{2}$ \\ ${ }^{1}$ Department of Cardiology, Zhejiang Province Hospital of Integrated Traditional Chinese \\ and Western Medicine, Hangzhou, Zhejiang $310003 ;{ }^{2}$ Department of Cardiology, Affiliated \\ Zhongshan Hospital of Dalian University, Dalian, Liaoning 116000, P.R. China
}

Received April 25, 2016; Accepted March 24, 2017

DOI: $10.3892 /$ etm.2017.5338

\begin{abstract}
Mice that lacked manganese-superoxide dismutase (Mn-SOD) activity exhibited the typical pathology of dilated cardiomyopathy (DCM). The aim of the present study was to investigate the effect of supplementation with omega-3 polyunsaturated fatty acids (n-3 PUFA) on heart function and oxidative stress biomarkers in mice with DCM. In the present study, heart/muscle-specific Mn-SOD-deficient mice $\left(\mathrm{H} / \mathrm{M}-\mathrm{Sod} 2^{-{ }^{-}}\right)$were treated with $\mathrm{n}-3$ PUFA $(30 \mathrm{mg} / \mathrm{kg} / \mathrm{day})$ for 10 weeks, and the reactive oxygen species (ROS) production in their heart mitochondria and cardiac function was subsequently assessed. n-3 PUFA treatment diminished ROS production and suppressed the progression of cardiac dysfunction. Furthermore, n-3 PUFA treatment effectively reversed the cardiac dysfunction and dilatation observed in symptomatic H/M-Sod2 ${ }^{--}$mice. Notably, n-3 PUFA treatment ameliorated a molecular defect in connexin 43. Hematoxylin-eosin staining indicated that the phenotype of DCM was also ameliorated following n-3 PUFA treatment. Furthermore, echocardiography demonstrated that cardiac function was significantly improved in the mice treated with $n-3$ PUFA $(\mathrm{P}<0.05)$. Meanwhile, pre-treatment with n-3 PUFA significantly decreased cardiomyocyte apoptosis $(\mathrm{P}<0.001)$. In conclusion, n-3 PUFA treatment is able to prevent murine DCM, primarily by reducing ROS production and improving myocardial apoptosis. Therefore, the impairment of ROS production is proposed as a potential therapy for DCM.
\end{abstract}

Correspondence to: Dr Qianxiao Li, Department of Cardiology, Zhejiang Province Hospital of Integrated Traditional Chinese and Western Medicine, 208 Ring Road, Hangzhou, Zhejiang 310003, P.R. China

E-mail: manyong160107@163.com

Key words: dilated cardiomyopathy, omega-3 polyunsaturated fatty acid, reactive oxygen species, apoptosis

\section{Introduction}

As a progressive heart muscle disease, dilated cardiomyopathy (DCM) is characterized by a dilated ventricular chamber, including impaired contraction of the left or both ventricles $(1,2)$. DCM typically leads to congestive heart failure, thereby enhancing morbidity and mortality among patients $(3,4)$. Several clinical studies have indicated that reactive oxygen species (ROS) are important in the pathogenesis of cardiovascular diseases, including DCM (5-7). Furthermore, it has been suggested that an imbalance between ROS production and antioxidant defenses may result in oxidative stress-related disorders, including DCM $(8,9)$. Epidemiological studies show that patients with cardiovascular diseases can be prevented from ROS injury through antioxidant reagents (10). Additionally, several potential sources of ROS have been proposed, including mitochondrial respiratory chain enzymes, xanthine oxidase, nicotinamide adenine dinucleotide phosphate (NADPH) oxidase and nitric oxide synthase (11). Abnormal ROS production may further result in aberrant morphological or functional changes, which are demonstrated as cardiac dysfunction (12).

Omega-3 polyunsaturated fatty acid (n-3 PUFA), a free radical scavenger, has long been suggested to be effective for combating oxidative stress (13-15). However, the specific mechanism through which n-3 PUFA protects the heart from DCM remains to be elucidated. Therefore, the present study aimed to determine the potential role of n-3 PUFA on DCM.

It has previously been indicated that the typical pathology of DCM is exhibited by mice lacking manganese-superoxide dismutase (Mn-SOD) activity $(16,17)$. Therefore, the present study used such mice as a DCM model to explore the effect of n-3 PUFA on the parameters of heart function and oxidative stress biomarkers in DCM mice.

\section{Materials and methods}

In vivo study protocols. A total of 8 male heart/muscle-specific Mn-SOD-deficient (H/M-Sod2 ${ }^{-/}$) mice (age, 8 weeks; weight, $22.5 \pm 3.1 \mathrm{~g}$ ) were purchased from The Fourth Affiliated Hospital of Harbin Medical University (Harbin, China). The mice were 
kept in a temperature- $\left(20-24^{\circ} \mathrm{C}\right)$ and humidity-controlled (45-55\%) environment, under a 12-h light/dark cycle, with free access to food and water. H/M-Sod2 ${ }^{-/-}$mice were fed with 3 mg/kg/day n-3 PUFA (n=4) (Sigma-Aldrich; Merck KGaA, Darmstadt, Germany) or vehicle $(n=4)$ (lauric acid, Sigma-Aldrich; Merck KGaA) (18) for 10 weeks, and cardiac function was evaluated by echocardiography. Mice were subsequently sacrificed, hearts were harvested and the proteins extracted. The present study was approved by the Zhejiang Province Hospital of Integrated Traditional Chinese and Western Medicine (Hangzhou, China).

Echocardiography. Two-dimensional (2D) echocardiography was performed with certain modifications according to previously reported methods (19). On the day of evaluation, sodium pentobarbital (50 mg/kg; P3761, Sigma-Aldrich; Merck KGaA) was administered by intraperitoneal injection to anesthetize the mice. After shaving the chest, 2D echocardiography was conducted with an echocardiographic system (model SSD-900; Hitachi-Aloka Medical, Ltd., Tokyo, Japan) and 7.5 MHz probe (UST-987-7.5; Hitachi-Aloka Medical, Ltd.). Furthermore, the fraction shortening (FS), ejection fraction (EF), left ventricular internal diameter (LVID) during systole and diastole, and endsystolic and end-diastolic volumes were calculated using Vevo Vasc Analysis software (version 2.2.3; Fujifilm VisualSonics, Inc., Toronto, Canada) as previously described (20).

Primary cardiomyocyte culture. Primary cardiomyocytes from mouse neonatal hearts were isolated as previously described (21). In brief, hearts were isolated and digested with collagenase type II solution (Worthington Biochemical Corporation, Lakewood, NJ, USA). Following digestion, the cells were plated in a $25-\mathrm{cm}^{2}$ culture flask for $2 \mathrm{~h}$ at $37^{\circ} \mathrm{C}$ in Dulbecco's modified Eagle's medium (DMEM; GE Healthcare Life Sciences, Logan, UT, USA) supplemented with $10 \%$ fetal bovine serum (Gibco; Thermo Fisher Scientific, Inc., Waltham, MA, USA) to collect cardiomyocytes. Attached cells were considered as non-myocytes and discarded, and the unattached cells were primarily cardiomyocytes.

Histological studies. For the histological analysis, heart tissues were fixed at room temperature for $10 \mathrm{~min}$ in $10 \%$ buffered formalin. Fixed tissues were subsequently dehydrated, embedded in paraffin, sectioned into $4-\mu \mathrm{m}$ slices and stained with hematoxylin and eosin (H\&E). Furthermore, Masson's trichrome was used to stain myocardial sections in order to evaluate the cardiomyocyte diameter and degree of fibrosis. Images were captured with a Pixera Pro600EX camera and a VANOX-S microscope (Olympus Corporation, Tokyo, Japan). Additionally, the fibrotic area and cardiomyocyte diameter ( $>30$ cells) were quantified with Qwin Plus V3 (Leica Microsystems, Inc., Buffalo Grove, IL, USA), and the collagen volume percentage was calculated as the mean of 5 fields for each animal.

Hoechst 33258 staining. Primary cardiomyocytes were cultured in 6 -well tissue culture plates $\left(1 \times 10^{5}\right.$ cells per well). The cells were incubated at $37^{\circ} \mathrm{C}$ in serum-free DMEM for $16 \mathrm{~h}$ at $70-80 \%$ confluence. The cells were then washed three times with cold PBS and fixed with 4\% formaldehyde (Zhongshan Technology Co., Ltd., Zhongshan, China) in PBS for $20 \mathrm{~min}$ at room temperature. Next, the cells were washed three times with cold PBS and stained with Hoechst 33258 (10 $\mu \mathrm{g} / \mathrm{ml} ; 50 \mu \mathrm{l} / \mathrm{slide}$; Sigma-Aldrich; Merck KGaA) at room temperature for $5 \mathrm{~min}$. Following staining, cold PBS was used to further rinse the cells, and were then examined under a fluorescence microscope.

Apoptosis assay. In order to detect the effects of n-3 PUFA on cell apoptosis, primary cardiomyocytes isolated from mice were washed with cold PBS three times. Next, flow cytometry was used to determine cell apoptosis with an Annexin-V fluorescein isothiocyanate-propidium iodide (FITC-PI) apoptosis kit (Invitrogen, Carlsbad, CA, USA). In summary, the cells $\left(1 \times 10^{6}\right)$ were washed with $1 \mathrm{X}$ PBS three times and suspended at $2-3 \times 10^{6}$ cells $/ \mathrm{ml}$ in X1 Annexin-V binding buffer [10 mM HEPES/NaOH, (pH 7.4), 140 mM NaCl, 2.5 mM $\mathrm{CaCl}_{2}$ ]. Annexin-V FITC and PI buffer were then added to the cells, which were then incubated at room temperature for $15 \mathrm{~min}$ in the dark. The cells that did not undergo any treatment were used as an internal control. Following incubation, the cells were filtered using a filter screen and analyzed using a flow cytometer within $1 \mathrm{~h}$ of staining. Cell apoptosis was analyzed using BD CellQuest Pro ${ }^{\mathrm{TM}}$ Analysis Software (BD Biosciences, San Jose, CA, USA).

Determination and quantification of ROS. Primary cardiomyocytes isolated from the mice were cultured on slides in a 6 -well chamber at $60 \%$ confluence at $37^{\circ} \mathrm{C}$. Two days later, the slides were washed with cold PBS three times. Additionally, the slides were treated with $5 \mu \mathrm{M}$ dihydroethidium (DHE; Vigorous Biotechnology Beijing Co., Ltd., Beijing, China) in serum-free DMEM F-12 medium (GE Healthcare Life Sciences) for $30 \mathrm{~min}$ at $37^{\circ} \mathrm{C}$ in darkness. Furthermore, the cells were fixed in $4 \%$ paraformaldehyde for $30 \mathrm{~min}$ at room temperature. The slides were then washed with cold PBS three times and mounted. Finally, immunofluorescence images were captured by fluorescence microscopy, and in order to quantify the intracellular ROS, the relative fluorescence intensities were analyzed using flow cytometry in the primary cardiomyocytes. Briefly, $1 \times 10^{6}$ cells were centrifuged $(200 \mathrm{x}$ g) for $10 \mathrm{~min}$ at room temperature and the supernatant was discarded. The cell pellet was resuspended in $1 \mathrm{ml}$ of PBS at room temperature. A $2 \mathrm{mM}$ solution of $\mathrm{H}_{2}$ DCFDA (Invitrogen; Thermo Fisher Scientific, Inc.) was freshly prepared in ethanol and $5 \mu \mathrm{l}$ was added to the cell suspension (final concentration $10 \mu \mathrm{M}$ ) and incubated at $37^{\circ} \mathrm{C}$ for $20 \mathrm{~min}$. Cells were centrifuged at $200 \mathrm{x} \mathrm{g}$ for $5 \mathrm{~min}$ at room temperature, the supernatant was discarded, and the cell pellet was resuspended in $500 \mu \mathrm{l}$ of PBS. Flow cytometric analysis was performed in duplicate using a flow cytometer and acquired data were analyzed using WinMDI v2.8 software (BD Biosciences).

Protein extraction, western blotting and antibodies. Cellular proteins were extracted using RIPA buffer $[50 \mathrm{mM}$ Tris $/ \mathrm{HCl}$, (pH 7.4), $150 \mathrm{mM} \mathrm{NaCl} 1 \%$ (v/v) NP-40,0.1\% (w/v) SDS; Beijing Solarbio Science \& Technology Co., Ltd., Beijing, China] with $0.3 \%$ (v/v) protease inhibitor (Sigma-Aldrich; Merck KGaA), $1 \%$ (v/v) phenylmethane sulfonyl fluoride (Beijing Solarbio 

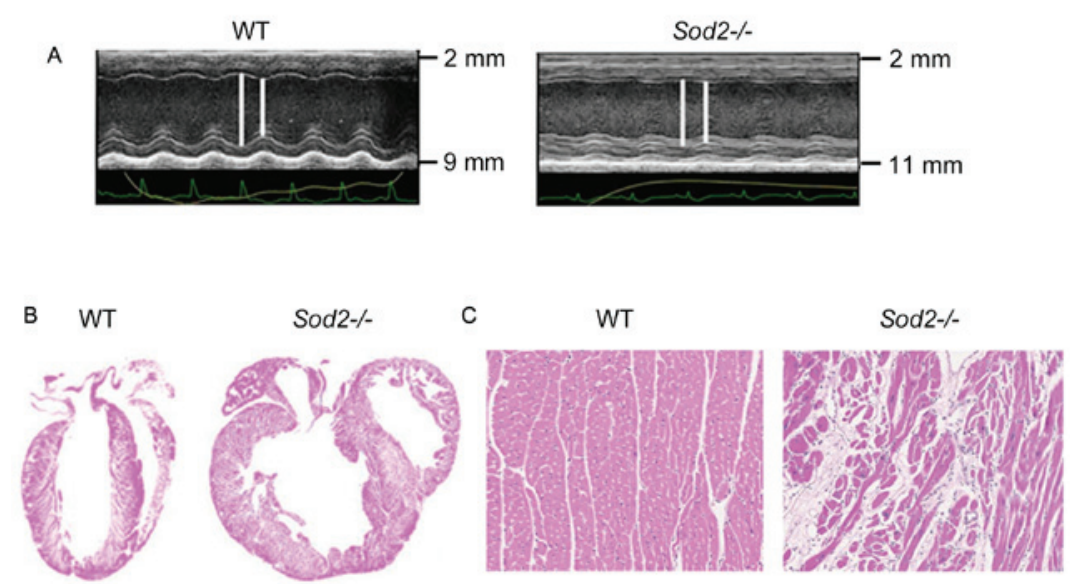

Figure 1. Cardiac dilation in hearts from H/M-Sod2 ${ }^{-/}$mice. (A) Hearts from H/M-Sod2 ${ }^{-}$mice demonstrated different parasternal long-axis M-mode echocardiographic images. (B) Hematoxylin \& eosin staining showed marked dilation of dilated cardiomyopathy hearts from H/M-Sod2/ mice compared with WT mice. (C) The myocardial sections from H/M-Sod2 $2^{--}$mice indicated eccentric hypertrophy and myocardial disarray compared with hearts from WT mice. H/M-Sod2 ${ }^{-/}$, heart/muscle-specific manganese-superoxide dismutase-deficient mice; WT, wild- type; PUFA, polyunsaturated fatty acids.

Science \& Technology Co., Ltd.) and $0.1 \%$ (v/v) phosphorylated proteinase inhibitor (Sigma-Aldrich, Inc.; Merck KGaA) for $15 \mathrm{~min}$ at room temperature. The lysates were collected for the total protein following centrifugation at $10,000 \mathrm{x} \mathrm{g}$ at $4^{\circ} \mathrm{C}$ for $15 \mathrm{~min}$. Furthermore, the bicinchoninic acid protein assay kit (Pierce; Thermo Fisher Scientific, Inc.) was used to determine the protein concentration. A total of $15 \mu \mathrm{g}$ protein per lane was separated by $10 \%$ SDS-PAGE and transferred onto a polyvinylidene difluoride membrane. After blocking with $8 \%(\mathrm{w} / \mathrm{v})$ milk in PBST for $2 \mathrm{~h}$ at room temperature, the membranes were then incubated with primary antibodies against GAPDH (cat. no. 2118; 1:5,000), cleaved-caspase 3 (cat. no. 9664; 1:1,000), p-JNK (cat. no. 4668; 1:1,000), JNK (cat. no. 9252; 1:1,000), p-NF-кB (cat. no. 3033; 1:1,000), NF-кB (cat. no. 8801; 1:1,000) (all Cell Signaling Technology, Inc., Danvers, MA, USA) and NOX4 (cat. no. ab109225; 1:1,000; Abcam, Cambridge, UK) overnight at $4^{\circ} \mathrm{C}$. Next, the membranes were washed with TBST three times. Additionally, they were incubated with horseradish peroxidase (HRP)-conjugated goat anti-rabbit or HRP-conjugated mouse anti-goat IgG (both 1:5,000; ZB-2301, Zhongshan Golden Bridge Biotechnology Co., Ltd., Beijing, China) for $2 \mathrm{~h}$ at room temperature and then washed three times with TBST. Enhanced chemiluminescence (EMD Millipore, Billerica, MA, USA) was used to determine the protein concentrations according to the manufacturer's recommendations. Finally, the relative contents of protein were normalized against GAPDH. All experiments were repeated three times. ImageJ $1.43 \mathrm{~b}$ software (National Institutes of Health, Bethesda, MD, USA) was used for densitometry analysis.

Determination of protein carbonylation and ATP content. The nuclear and mitochondrial fractions of the heart were isolated in order to quantify cardiac protein carbonylation. In brief, the crude nuclear fractions were isolated from tissue homogenates at $1,000 \mathrm{x}$ g for $5 \mathrm{~min}$ at $4^{\circ} \mathrm{C}$ and washed with PBS at $4^{\circ} \mathrm{C}$. The mitochondrial fractions were separated as previously described (22). The carbonylation of mitochondrial and nuclear protein was evaluated using an Oxyblot protein oxidation detection kit (EMD Millipore) according to the manufacturer's instructions. Immunoreactive spots were visualized with enhanced chemiluminescence (GE Healthcare, Buckinghamshire, UK) and quantified with ImageJ 1.43b (National Institutes of Health, Bethesda, MD, USA). Furthermore, the ATP content was determined using an ATP assay kit (colorimetric/fluorometric) (cat. no. ab83355; Abcam), according to the manufacturer's instructions.

Statistical analysis. Data are presented as the mean \pm standard error from three independent experiments. Statistical analysis was performed with Student's-test. $\mathrm{P}<0.05$ was considered to indicate a statistically significant difference.

\section{Results}

Cardiac dilation in hearts from H/M-Sod $2^{-/}$mice. Compared with wild-type (WT) mice, hearts from $\mathrm{H} / \mathrm{M}-\mathrm{Sod}^{-/-}$mice yielded increased parasternal long-axis M-mode echocardiographic images (Fig. 1A). As shown in Fig. 1B, H\&E staining revealed an evident dilation of DCM hearts from $\mathrm{H} / \mathrm{M}-\mathrm{Sod} 2^{-/-}$mice compared with those from WT mice. Furthermore, the myocardial sections from $\mathrm{H} / \mathrm{M}-\mathrm{Sod} 2^{--}$mice indicated eccentric hypertrophy and myocardial disarray compared with hearts from WT mice (Fig. 1C). These data showed the cardiac dilation phenotype in hearts from $\mathrm{H} / \mathrm{M}-\mathrm{Sod} 2^{-/-}$mice.

n-3 PUFA ameliorates cardiac enlargement in H/M-Sod2-1mice. To identify whether n-3 PUFA prevents cardiac enlargement in $\mathrm{H} / \mathrm{M}-\mathrm{Sod} 2^{-/-}$mice, Masson's trichrome staining demonstrated that interstitial fibrosis was markedly improved after n-3 PUFA treatment, suggesting that n-3 PUFA was able to partially reverse cardiac fibrosis in the H/M-Sod 2-- mice (Fig. 2A). Furthermore, the cardiomyocyte diameter was significantly decreased in $\mathrm{H} / \mathrm{M}-\mathrm{Sod}_{2}{ }^{--}$mice treated with n-3 PUFA, compared with untreated mice, indicating that cardiac hypertrophy was ameliorated by $n-3$ PUFA treatment (Fig. 2B). Following n-3 PUFA treatment, the percentage of FS was significantly enhanced, compared with untreated mice (Fig. 2C). These data suggested that 

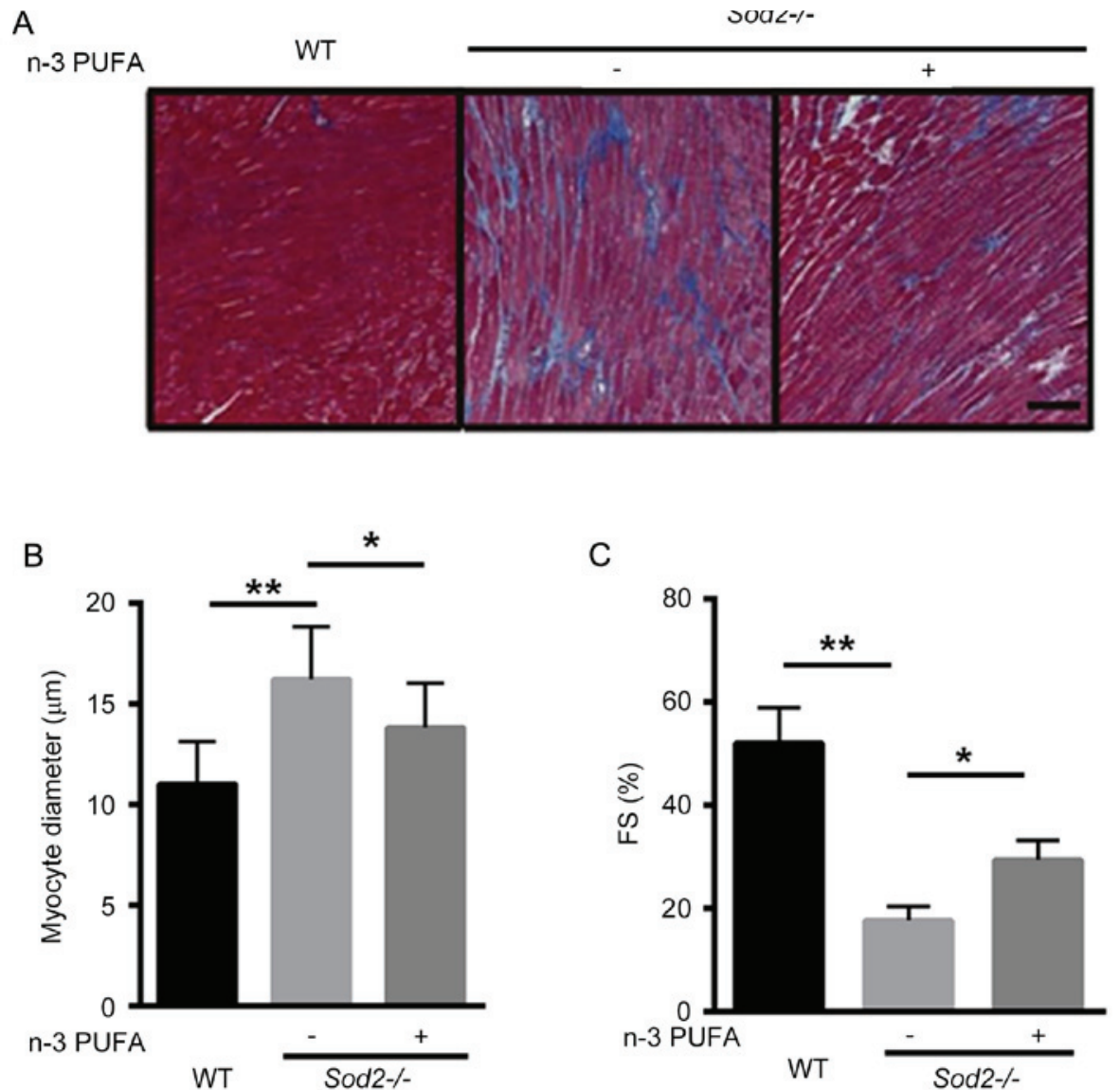

Figure 2. $\mathrm{n}-3$ PUFA prevents cardiac enlargement in H/M-Sod2 $2^{-/}$mice. (A) Masson's trichrome staining demonstrated that interstitial fibrosis was markedly ameliorated following n-3 PUFA treatment. (B) Cardiomyocyte diameter was significantly decreased in the H/M-Sod $2^{-/}$mice treated with n-3 PUFA, compared with untreated mice. (C) Following n-3 PUFA treatment, FS was significantly increased. $\mathrm{n}=5$; ${ }^{*} \mathrm{P}<0.05$; ${ }^{* *} \mathrm{P}<0.01$. $\mathrm{n}-3$ PUFA, omega-3 polyunsaturated fatty acids; H/M-Sod2-/- heart/muscle-specific manganese-superoxide dismutase-deficient mice; FS, fraction shortening; WT, wild-type.
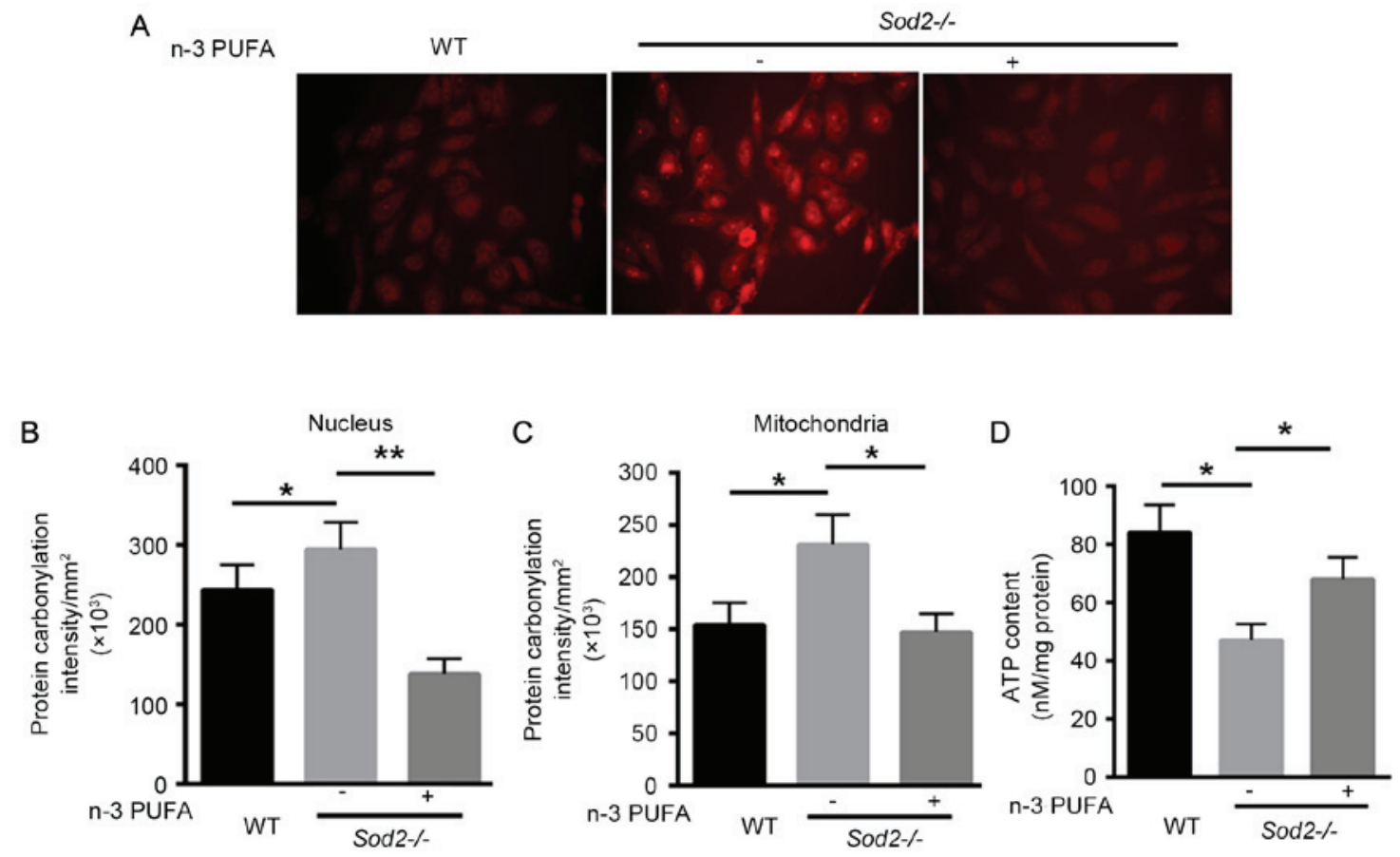

Figure 3. n-3 PUFA decreases ROS production in H/M-Sod2/- mice. (A) Dihydroethidium staining demonstrated that n-3 PUFA treatment markedly decreased ROS production in primary cardiomyocytes isolated from $\mathrm{H} / \mathrm{M}-\mathrm{Sod} 2^{--}$. Protein carbonylation was significantly decreased in (B) the nuclei and (C) mitochondria of primary cardiomyocytes treated by n-3 PUFA of H/M-Sod2 ${ }^{--}$mice. (D) n-3 PUFA treatment recovered $21 \%$ more ATP content compared with the control H/M-Sod2 ${ }^{-\digamma}$ mice. $n=5 ;{ }^{*} \mathrm{P}<0.05 ;{ }^{* *} \mathrm{P}<0.01$ vs. WT. $\mathrm{n}-3$ PUFA, omega-3 polyunsaturated fatty acids; ROS, reactive oxygen species; H/M-Sod2 ${ }^{-1}$, heart/muscle-specific manganese-superoxide dismutase-deficient mice; WT, wild-type. 
A

n-3 PUFA

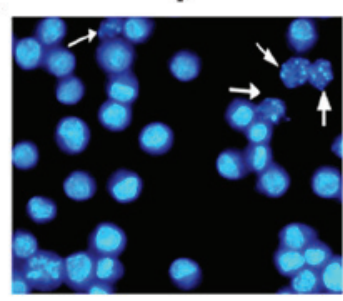

B

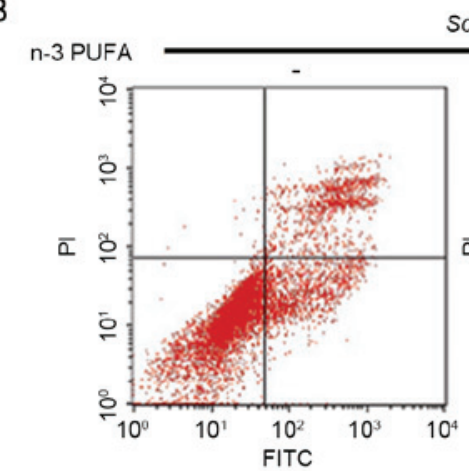

Sod $2-\%$

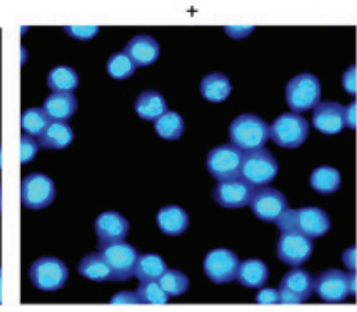

Sod $2-/-$

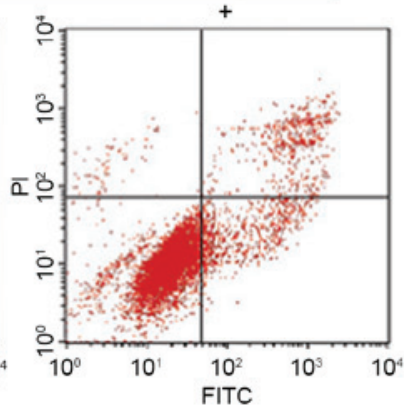

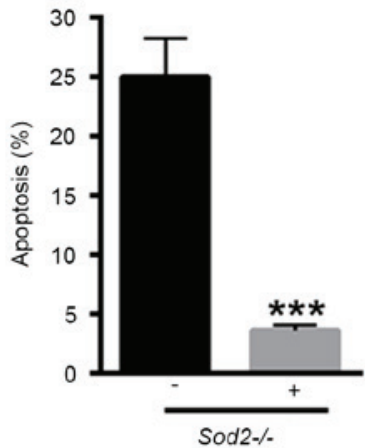

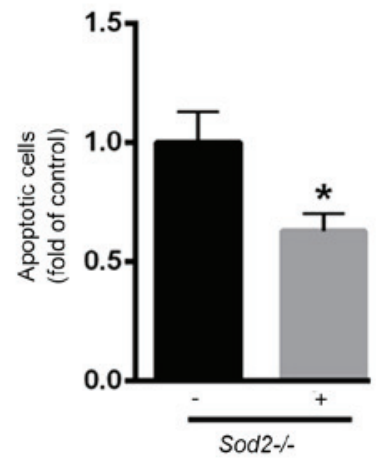

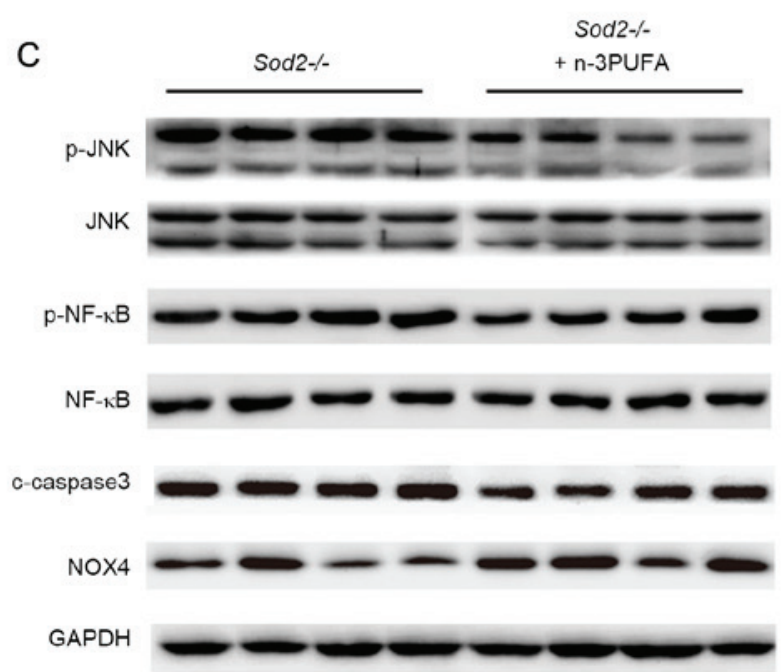

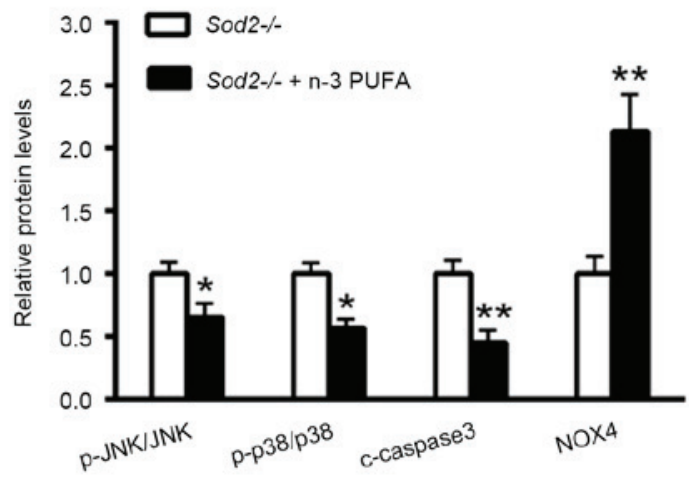

Figure 4. n-3 PUFA reduces primary cardiomyocyte apoptosis. (A) Hoechst staining (white arrows indicate apoptotic cells) and (B) flow cytometry were applied to determine apoptosis of primary cardiomyocytes isolated from H/M-Sod2 $2^{--}$mice. (C) Western blot analysis of the changed protein patterns after n-3 PUFA treatment in the hearts of $\mathrm{H} / \mathrm{M}-\mathrm{Sod} 2{ }^{-/}$mice. $\mathrm{n}=5 ;{ }^{*} \mathrm{P}<0.05,{ }^{* *} \mathrm{P}<0.01$ and ${ }^{* * * *} \mathrm{P}<0.001$ vs. WT mice. $\mathrm{n}-3$ PUFA, omega-3 polyunsaturated fatty acids; H/M-Sod $2^{-/}$, heart/muscle-specific manganese-superoxide dismutase-deficient mice; WT, wild-type; FITC, fluorescein isothiocyanate; PI, propidium iodide; JNK, c-Jun N-terminal kinases; NF, nuclear factor; c, cleaved; NOX4, nicotinamide adenine dinucleotide phosphate oxidase 4.

n-3 PUFA treatment ameliorated cardiac dysfunction in $\mathrm{H} / \mathrm{M}-\mathrm{Sod} 2^{-/-}$mice.

n-3 PUFA decreases ROS production in H/M-Sod2 ${ }^{-/-}$mice. The ROS production in primary cardiomyocytes isolated from $\mathrm{H} / \mathrm{M}-\mathrm{Sod} 2^{-/}$and WT mice was identified. As shown in Fig. 3A, DHE staining revealed that n-3 PUFA treatment markedly decreased the ROS production in primary cardiomyocytes isolated from H/M-Sod2 ${ }^{-/}$. Subsequently, oxidative protein damage was investigated in nuclei and mitochondria. Protein carbonylation was significantly decreased in the nuclei and mitochondria of primary cardiomyocytes of H/M-Sod2 ${ }^{-/}$mice treated with n-3 PUFA (Fig. 3B and C).
Furthermore, n-3 PUFA treatment recovered $21 \%$ more ATP content compared with the untreated H/M-Sod $2^{-/-}$mice (Fig. 3D). These data indicated that n-3 PUFA decreases ROS generation, protein damage and the ATP content in $\mathrm{H} / \mathrm{M}-\mathrm{Sod} 2^{-/-}$mice.

n-3 PUFA reduces primary cardiomyocyte apoptosis. In order to investigate the potential role of n-3 PUFA on cell apoptosis, Hoechst staining and flow cytometry were applied to determine apoptosis of primary cardiomyocytes isolated from H/M-Sod $2^{-/}$mice. Following n-3 PUFA treatment, primary cardiomyocytes isolated from $\mathrm{H} / \mathrm{M}-\mathrm{Sod} 2^{-/-}$mice after apoptosis were evidently decreased compared with the 
untreated group (Fig. 4A and B). Notably, the phosphorylation levels of c-Jun N-terminal kinases (JNK), nuclear factor (NF) $-\kappa \mathrm{B}$ and cleaved-caspase 3 were significantly decreased with n-3 PUFA treatment, whereas the expression of NADPH oxidase 4 (NOX4) was significantly increased (Fig. 4C).

\section{Discussion}

The present study identified that n-3 PUFA treatment was able to partially abolish cardiac enlargement and dysfunction in $\mathrm{H} / \mathrm{M}-\mathrm{Sod} 2^{-/}$mice and that the protective effects of n-3 PUFA predominantly originated from reduced ROS production and cardiomyocyte apoptosis. Together, these data indicate that decreased oxidative damage contributes to the reduction of cardiac enlargement in $\mathrm{H} / \mathrm{M}-\mathrm{Sod} 2^{-/-}$mice.

Knockout of the Mn-SOD gene in the heart and muscle may lead to cardiac oxidative stress, which leads to contractile dysfunction, fibrosis and myocyte damage (23). According to echocardiographic analysis, hearts from H/M-Sod2 ${ }^{-/-}$mice were significantly enlarged. Furthermore, EF and FS were also found to be reduced in the hearts of $\mathrm{H} / \mathrm{M}-\mathrm{Sod} 2^{-/-}$mice, indicating severe DCM and cardiac dysfunction of these mice. Notably, n-3 PUFA treatment was demonstrated to improve histological abnormalities in DCM hearts of H/M-Sod2 ${ }^{-1-}$ mice, such as fibrosis, compared with hearts from the control group. This observation is in accordance with the Masson's trichrome-stained section analysis and suggests that fibrosis is improved in DCM hearts.

ROS are widely associated with age-related diseases, such as Alzheimer's and Parkinson's disease, and heart failure $(24,25)$. During mitochondrial respiration, small amounts of mitochondrial ROS production may be cleared by scavenging systems. In the presence of NOX4, superoxide anions were significantly decreased in dilated cardiomyopathy, suggesting the protective role of NOX4 on ROS production in $\operatorname{HF}(26,27)$. In accordance with the above observations, n-3 PUFA treatment was found to significantly increase the protein level of NOX4 in DCM hearts of $\mathrm{H} / \mathrm{M}-\mathrm{Sod} 2^{-/-}$mice, suggesting the protective role of n-3 PUFA in DCM. These results indicate that mitochondrial dysfunction may be improved by n-3 PUFA treatment in DCM.

It is widely reported that abnormal ROS production and apoptosis are important in the pathology of DCM $(28,29)$. Therefore, to prevent diabetic cardiomyopathy, it is important to simultaneously inhibit oxidative stress and apoptosis. The present study explored the effects of n-3 PUFA on cardiomyocyte apoptosis. It was observed that n-3 PUFA treatment led to a significant reduction of cell apoptosis in primary cardiomyocytes isolated from H/M-Sod2-- mice compared with the untreated group. Accordingly, the protein level of cleaved-caspase 3 was significantly decreased following n-3 PUFA treatment. Furthermore, it was also shown that n-3 PUFA demonstrated anti-inflammatory effects since the phosphorylation levels of JNK and NF- $\kappa$ B were significantly decreased.

In conclusion, oxidative stress was shown to increase in the DCM model of H/M-Sod2 ${ }^{-/}$mice. Notably, the results indicate that n-3 PUFA may be used as an antioxidant to protect hearts from DCM, primarily by reducing ROS production and cardiomyocyte apoptosis. Finally, the present study may assist in the development of a novel therapy and prevention for DCM in human patients.

\section{References}

1. Arumugam S, Thandavarayan RA, Veeraveedu PT, Nakamura T, Arozal W, Sari FR, Giridharan VV, Soetikno V, Palaniyandi SS, Harima M, et al: Beneficial effects of edaravone, a novel antioxidant, in rats with dilated cardiomyopathy. J Cell Mol Med 16: 2176-2185, 2012.

2. Elliott PM: Classification of cardiomyopathies: Evolution or revolution? J Am Coll Cardiol 62: 2073-2074, 2013.

3. Cecchi F, Tomberli B and Olivotto I: Clinical and molecular classification of cardiomyopathies. Glob Cardiol Sci Pract 2012: 4, 2012.

4. Elliott P, Andersson B, Arbustini E, Bilinska Z, Cecchi F, Charron P, Dubourg O, Kühl U, Maisch B, McKenna WJ, et al: Classification of the cardiomyopathies: A position statement from the European society of cardiology working group on myocardial and pericardial diseases. Eur Heart J 29: 270-276, 2008.

5. Lynch TL IV, Sivaguru M, Velayutham M, Cardounel AJ, Michels M, Barefield D, Govindan S, dos Remedios C, van der Velden J and Sadayappan S: Oxidative stress in dilated cardiomyopathy caused by MYBPC 3 mutation. Oxid Med Cell Longev 2015: 424751, 2015.

6. Samarghandian S, Farkhondeh T and Samini F: Honey and health: A review of recent clinical research. Pharmacognosy Res 9: 121-127, 2017.

7. Zhang P, Yi LH, Meng GY, Zhang HY, Sun HH and Cui LQ: Apelin-13 attenuates cisplatin-induced cardiotoxicity through inhibition of ROS-mediated DNA damage and regulation of MAPKs and AKT pathways. Free Radic Res 51: 449-459, 2017.

8. Kono Y, Nakamura K, Kimura H, Nishii N, Watanabe A, Banba K, Miura A, Nagase S, Sakuragi S, Kusano KF, et al: Elevated levels of oxidative DNA damage in serum and myocardium of patients with heart failure. Circ J 70: 1001-1005, 2006.

9. Gopal DM and Sam F: New and emerging biomarkers in left ventricular systolic dysfunction-insight into dilated cardiomyopathy. J Cardiovasc Transl Res 6: 516-527, 2013.

10. White M, Ducharme A, Ibrahim R, Whittom L, Lavoie J, Guertin MC, Racine N, He Y, Yao G, Rouleau JL, et al: Increased systemic inflammation and oxidative stress in patients with worsening congestive heart failure: Improvement after short-term inotropic support. Clin Sci (Lond) 110: 483-489, 2006.

11. Willcox BJ, Curb JD and Rodriguez BL: Antioxidants in cardiovascular health and disease: Key lessons from epidemiologic studies. Am J Cardiol 101: 75D-86D, 2008.

12. Firuzi O, Miri R, Tavakkoli M and Saso L: Antioxidant therapy: Current status and future prospects. Curr Med Chem 18: 3871-3888, 2011.

13. Rizos EC and Elisaf M: Omega-3 supplements and cardiovascular disease. Re: Sperling LS, Nelson JR. History and future of omega-3 fatty acids in cardiovascular disease. Curr Med Res Opin 2015: 32(2);301-311. Curr Med Res Opin 32: 583-584, 2016.

14. Sperling LS and Nelson JR: History and future of omega-3 fatty acids in cardiovascular disease. Curr Med Res Opin 32: 301-311, 2016.

15. Colussi G, Catena C and Sechi LA: $\omega-3$ Polyunsaturated fatty acids effects on the cardiometabolic syndrome and their role in cardiovascular disease prevention: An update from the recent literature. Recent Adv Cardiovasc Drug Discov 9: 78-96, 2014.

16. Kawakami S, Matsuda A, Sunagawa T, Noda Y, Kaneko T, Tahara S, Hiraumi Y, Adachi S, Matsui H, Ando K, et al: Antioxidant, EUK-8, prevents murine dilated cardiomyopathy. Circ J 73: 2125-2134, 2009.

17. Shimizu T, Nojiri H, Kawakami S, Uchiyama S and Shirasawa T: Model mice for tissue-specific deletion of the manganese superoxide dismutase gene. Geriatr Gerontol Int 10 (Suppl 1): S70-S79, 2010.

18. Fujita N, Hiroe M, Ohta Y, Horie T and Hosoda S: Chronic effects of metoprolol on myocardial beta-adrenergic receptors in doxorubicin-induced cardiac damage in rats. J Cardiovasc Pharmacol 17: 656-661, 1991.

19. Cittadini A, Strömer H, Katz SE, Clark R, Moses AC, Morgan JP and Douglas PS: Differential cardiac effects of growth hormone and insulin-like growth factor-1 in the rat. A combined in vivo and in vitro evaluation. Circulation 93: 800-809, 1996.

20. Shen T, Aneas I, Sakabe N, Dirschinger RJ, Wang G, Smemo S, Westlund JM, Cheng H, Dalton N, Gu Y, et al: Tbx 20 regulates a genetic program essential to adult mouse cardiomyocyte function. J Clin Invest 121: 4640-4654, 2011. 
21. Ieda M, Tsuchihashi T, Ivey KN, Ross RS, Hong TT, Shaw RM and Srivastava D: Cardiac fibroblasts regulate myocardial proliferation through beta1 integrin signaling. Dev Cell 16: 233-244, 2009.

22. Van Remmen H, Williams MD, Guo Z, Estlack L, Yang H, Carlson EJ, Epstein CJ, Huang TT and Richardson A: Knockout mice heterozygous for Sod2 show alterations in cardiac mitochondrial function and apoptosis. Am J Physiol Heart Circ Physiol 281: H1422-H1432, 2001.

23. Dayal S, Baumbach GL, Arning E, Bottiglieri T, Faraci FM and Lentz SR: Deficiency of superoxide dismutase promotes cerebral vascular hypertrophy and vascular dysfunction in hyperhomocysteinemia. PLoS One 12: e0175732, 2017.

24. Klein JA and Ackerman SL: Oxidative stress, cell cycle, and neurodegeneration. J Clin Invest 111: 785-793, 2003.

25. Ide T, Tsutsui H, Kinugawa S, Suematsu N, Hayashidani $S$, Ichikawa K, Utsumi H, Machida Y, Egashira K and Takeshita A: Direct evidence for increased hydroxyl radicals originating from superoxide in the failing myocardium. Circ Res 86: 152-157, 2000.
26. Ago T, Matsushima S, Kuroda J, Zablocki D, Kitazono T and Sadoshima J: The NADPH oxidase Nox4 and aging in the heart. Aging (Albany NY) 2: 1012-1016, 2010

27. Kuroda J, Ago T, Matsushima S, Zhai P, Schneider MD and Sadoshima J: NADPH oxidase 4 (Nox4) is a major source of oxidative stress in the failing heart. Proc Natl Acad Sci USA 107: 15565-15570, 2010.

28. Zhang F, Lin X, Yu L, Li W, Qian D, Cheng P, He L, Yang H and Zhang C: Low-dose radiation prevents type 1 diabetes-induced cardiomyopathy via activation of AKT mediated anti-apoptotic and anti-oxidant effects. J Cell Mol Med 20: 1352-1366, 2016.

29. Yu H, Zhen J, Yang Y, Gu J, Wu S and Liu Q: Ginsenoside Rg1 ameliorates diabetic cardiomyopathy by inhibiting endoplasmic reticulum stress-induced apoptosis in a streptozotocin-induced diabetes rat model. J Cell Mol Med 20: 623-631, 2016. 